\title{
Motivos que llevan a realizar actividad física en personas adultas del estado de Sonora, México
}

\author{
Motives that lead to physical activity in adults in the state of Sonora, Mexico \\ Graciela Hoyos Ruiz ${ }^{1}$ \\ Alejandrina Bautista Jacobo ${ }^{2}$ \\ María Elena Chávez Valenzuela ${ }^{3}$ \\ Gricelda Henry Mejía ${ }^{4}$ \\ Manuel Alejandro Vázquez Bautista ${ }^{5}$
}

Fecha de recepción: 2-2-2019

Fecha de aprobación: 3-02-2020

\begin{abstract}
Resumen
El objetivo de este trabajo fue reportar los principales motivos por los cuales una población adulta de Sonora, México, realiza actividad física, tomando en cuenta el sexo y edad. Se llevó a cabo un estudio transversal descriptivo en una muestra por conveniencia de 614 personas en un rango de edad de 18 a 74 años, que realizaban actividad física en diferentes centros deportivos y en algunos gimnasios reconocidos de la ciudad. Para lograr el objetivo, se utilizó como instrumento de medición la escala validad en versión castellano de Motives for Physical Activities Measure-Revised (MPAM-R) que agrupa cinco motivos por los cuales las personas realizan actividad física: disfrute, apariencia, social, fitness/salud y competencia. Además de las preguntas de la encuesta, se solicitó información sobre el tiempo que llevan practicando la actividad física, frecuencia semanal y horas que le dedican al día. Se concluye de este estudio que los motivos más importantes para hacer actividad física tanto en hombres como en las mujeres encuestadas son la salud y el disfrute por la actividad, y lo que menos los motiva son las relaciones sociales, como la convivencia con los amigos.
\end{abstract}

Palabras claves: Motivación, actividad física, fitness/salud, competencia, apariencia.

\footnotetext{
1 Docente de la División de Ciencias Biológicas y de la Salud, Departamento de Ciencias del Deporte y la Actividad Física, Universidad de Sonora, México. Correo electrónico: ghoyos@guaymas.uson.mx

2 Docente de Bioestadística del Departamento de Ciencias del Deporte y la Actividad Física, Universidad de Sonora, México. Correo electrónico: ale@mat.uson.mx

3 Docente del Departamento de Ciencias del Deporte y la Actividad Física, Universidad de Sonora, México. Correo electrónico: malenamanzana@ gmail.com

4 Docente del Departamento de Ciencias del Deporte y la Actividad Física, Universidad de Sonora, México. Correo electrónico: grisyhenry@ hotmail.com

5 Estudiante de posgrado en el Centro de Investigación en Alimentación y Desarrollo, Universidad de Sonora, México. Correo electrónico: vazquez.bautista@gmail.com.
} 


\begin{abstract}
The objective of this paper was to report the main reasons why an adult population in Sonora, Mexico engages in physical activity considering their sex and age. A cross-sectional descriptive study was conducted on a convenience sample of 614 people in the 18-74 age range who engaged in physical activity in different sports centers and in some of the city's recognized gyms. In order to achieve the objective, the valid scale in Spanish version of Motives for Physical Activities Measure-Revised (MPAM-R) was used as a measurement instrument. This scale groups five reasons why people engage in physical activity: enjoyment, appearance, social, fitness/health and competition. In addition to the survey questions, information was requested on how long they have been doing physical activity, weekly frequency and the hours they devote to it each day. It is concluded from this study that the most important reasons for doing physical activity in both men and women surveyed are health and enjoyment of activity, and what least motivates them are social relationships, such as living with friends.
\end{abstract}

Keywords: Motivation, physical activity, fitness/health, competition, appearance.

\title{
I. Introducción
}

En los últimos años, en México, se han reportado investigaciones sobre la actividad física (AF) y la importancia que esta tiene para la salud en diferentes poblaciones (Cruz, Salazar \& Cruz, 2013; Zamarripa et al., 2013; Muñoz et al., 2016). Por su parte, el Instituto Nacional de Estadística y Geografía, en el año 1996, recopila por primera vez información relacionada con el nivel de actividad física con el Módulo de Práctica Deportiva y Ejercicio Físico (MOPRADEF) para personas de 15 y más años (INEGI, 2018). También, en el último informe registrado por MOPRADEF, destaca que las principales razones por las cuales la población no realiza actividad física o deporte son: por falta de tiempo (45.8\%), cansancio por el trabajo (24.8\%) y problemas de salud (18.2\%). Según datos, a finales del 2018, el 41.7\% de la población mexicana de 18 años en adelante realiza deporte o ejercicio físico en su tiempo libre (INEGI, 2018).

Realizar actividad física aumenta la calidad de vida y contribuye a mejorar la salud de las personas (Secretary of Health and Human Services [HHS], 2018), mientras que el sedentarismo es uno de los principales factores de riesgo asociados con el aumento de peso y, por tanto, de obesidad. Esta última se ha convertido en un serio problema de salud pública, por sus repercusiones en el aumento de enfermedades no transmisibles como la diabetes, cáncer o problemas cardiovasculares y cabe destacar que en México, solamente en 2013, murieron 57 mil personas a causas de estos padecimientos (Barquera, 2013).
Según datos del Instituto Nacional de Estadística y Geografía, más de la mitad de la población adulta es físicamente inactiva (INEGI, 2016) y el 72.5\% presenta sobrepeso u obesidad (ENSANUT, 2016). Como consecuencia, en México se han implementado programas para motivar a las personas a realizar AF, como el Programa Nacional de Cultura Física y Deporte, que en el 2012 superó seis veces la meta lograda en el 2006 de 5.1 millones de personas involucradas en actividades físicas regularmente; sin embargo, según los últimos registros aportados por INEGI, más del $50 \%$ de la población adulta es sedentaria (INEGI, 2018).

Los motivos por los cuales las personas realizan o no $\mathrm{AF}$ pueden ser diversos y depender de la edad y del grupo de la población a la que se pertenece (Moreno \& Cruz, 2015; Pérez \& Sánchez, 2015), y a sugerencia de algunos autores, conocerlos puede favorecer al diseño de estrategias efectivas para disminuir el sedentarismo y el problema de sobrepeso u obesidad en las poblaciones (Sanclemente \& Arias, 2014). Por ejemplo, en las mujeres el principal motivo para realizar ejercicio es la estética, cuidar su figura y mantener un peso adecuado (Tylka \& Homan, 2015), así como mantener un buen estado de salud y la recreación (Cambronero et al., 2015; Moreno, et al., 2016). En cambio, en poblaciones masculinas se ha detectado que el motivo principal para la AF es mejorar sus habilidades y la competencia personal (Guedes et al., 2013). 
Para algunos autores, la motivación es un aspecto psicológico regulador del buen desempeño de cierta actividad, ya sea física o de cualquier ámbito de la sociedad (Valdés, 1996). Así también, esta puede ser de carácter intrínseca y extrínseca, donde la primera consiste en desempeñar una actividad por placer y satisfacción sin esperar recompensa alguna, mientras que la segunda provoca realizar una actividad para recibir un reconocimiento ya sea material o social, tener una buena imagen ante los demás o sentirse aceptado por los amigos, por ejemplo (Deci \& Ryan, 1985). Dada las cifras alarmantes de deserción a la AF en algunas poblaciones, conocer los motivos por los cuales la realizan, si lo hacen, puede contribuir al diseño de estrategias orientadas a poblaciones específicas con el fin de promover esta práctica de manera constante.

En el caso de México, se encuentra publicado en el Diario Oficial el Programa Nacional de Cultura Física y Deporte, en el cual se encuentran integrados los objetivos, estrategias y líneas de acción que permitirán incorporar a la población en general a la práctica de la actividad física y deportiva. El primer objetivo que se menciona es lograr aumentar la práctica del "deporte social" para mejorar el bienestar de la población. Para lograrlo, una de las estrategias que se ha implementado es el programa "Ponte al 100" en todos los municipios de más de 15,000 habitantes para combatir el sedentarismo (CONADE, 2016).

Actualmente, la información sobre los motivos por los cuales la población mexicana se ejercita es escasa. Se tiene como dato el estudio realizado por Muñoz et al. (2016), en el cual identifica los principales motivos por los que una población mexicana en edades entre 6 y 90 años hace actividad física. Aclarado esto, el objetivo de este trabajo es indagar las motivaciones por las cuales el adulto mexicano, específicamente en Sonora, realiza AF, y así determinar si existen diferencias por sexo, edad y tiempo dedicado.

\section{Metodología}

Para la metodología se llevó a cabo un estudio transversal descriptivo en una población de personas que realizaba actividad física. Para determinar los participantes, se tomó una muestra a conveniencia de 614 personas que realizaban actividad física, todos de la localidad de Hermosillo, Sonora. Como criterio de inclusión, se consideró tener 18 años o más y firmar el formato de consentimiento informado.

Por otra parte, el instrumento utilizado fue la versión en español de la escala Motives for Physical Activities Measure-Revised (MPAM-R), de Moreno, Cervelló y Martínez (2007). Esta escala mide los motivos para la práctica de la actividad física mediante 30 ítems policotómicos en una escala tipo Likert de siete puntos: Totalmente en desacuerdo (1), en desacuerdo (2), ligeramente en desacuerdo (3), neutro (4), ligeramente de acuerdo (5), de acuerdo (6) y totalmente de acuerdo (7). De acuerdo con ello, a cada respuesta se le asigna un valor numérico. Así, el participante obtiene una puntuación respecto a su respuesta, y al final, su puntaje total, el cual es calculado sumando las puntuaciones obtenidas en relación con todas las afirmaciones. De esta manera, cuanto más favorable o más de acuerdo sea la opinión de un sujeto, mayor será la puntuación obtenida en la escala, y cuanto más desfavorable o en desacuerdo sea la opinión de este, menor será la puntuación que obtendrá.

La escala MPAM-R agrupa cinco factores o subescalas: disfrute, apariencia, social, fitness/salud y competencia. La validación de la escala fue hecha en una población española con niveles de confiabilidad en su mayoría con valores por arriba de 0.80 . En disfrute, se reportó un $\alpha=0.84$; apariencia con $\alpha=0.87$; social $\alpha=0.81$; fitness/salud con $\alpha=0.80$ y competencia con $\alpha=0.85$. Los ítems del factor disfrute y competencia implican una motivación intrínseca del sujeto, mientras que los de la escala social, fitness/salud y apariencia contiene aspectos de la motivación extrínseca.

$\mathrm{Al}$ instrumento se le agregaron algunas preguntas de identificación como sexo, edad, escolaridad e información laboral. Se registró además las variables de peso y 
estatura, ambas percibidas por el participante. La edad se agrupó en cinco categorías, según la clasificación de la INEGI para su comparación: $1=18$ a 24; $2=25$ a 34; 3 $=35$ a 44; $4=45$ a 54 y $5=55$ y más. Como información adicional, se agregaron a la escala algunas preguntas, formuladas por los propios investigadores, sobre el tiempo, días y horas de la práctica de actividad física agrupadas de manera categórica.

En cuanto al procedimiento, el instrumento se aplicó a personas que asisten a centros deportivos al aire libre como La Milla de la Universidad de Sonora; en los alrededores del gimnasio Ana Gabriela Guevara y la pista para caminar-trotar ubicada en el boulevard Progreso así como en algunos gimnasios reconocidos de la ciudad. Antes de aplicar el instrumento, se dio una breve explicación de los objetivos del estudio y se les preguntó la edad para determinar si la persona cumplía los criterios de inclusión. A las personas interesadas en participar se les solicitó firmar la carta de consentimiento informado. El tiempo promedio de aplicar y contestar el instrumento fue de 25 minutos.

Ahora bien, para el análisis estadístico se utilizó el software SPSS V. 21. Se presentan a su vez tablas de frecuencia en porcentajes para las variables categóricas, así como algunas medidas descriptivas como la media y desviación estándar para las variables numéricas. Para obtener el puntaje medio de cada ítem se sumaron los valores obtenidos en la respuesta para todos los encuestados, y se dividió sobre el total de la muestra (n $=614$ ). El puntaje promedio de cada factor se obtuvo de la misma forma, solo que tomando en cuenta los ítems del factor.

Para valorar la fiabilidad de la escala se utilizó el coeficiente de consistencia interna Alpha de Cronbach que asume que una escala es fiable cuando la variabilidad de las puntuaciones observadas es atribuible a las diferencias existentes entre los sujetos. Cabe destacar que para propósitos investigativos son aceptables valores mayores o iguales a 0.70 (George \& Mallery, 2003).
Se utilizó la prueba de la Chi-cuadrada para comprobar la relación entre las variables categóricas. En algunos casos se utilizó el coeficiente tau-b de Kendall, para medir la asociación en variables ordinales. Dado el incumplimiento de normalidad realizado con la prueba de KolmogorovSmirnov, se utilizaron las pruebas no paramétricas U de Mann-Whitney y Kruskal-Wallis para detectar diferencias significativas entre los grupos de los puntajes medios de cada factor. Para la comparación múltiple se procedió a calcular la prueba de Tukey. El nivel de significancia usado fue de 0.05 .

\section{Resultados}

\subsection{Características generales de la población de estudio}

La muestra estaba conformada en su mayoría por mujeres (50.71\%), mientras que el rango de edad de los participantes fue de 18 a 74 años (27.93 \pm 10.49$)$. Por otro lado, un $56.7 \%$ de los encuestados mencionó que su nivel de estudio es licenciatura y sólo un 2\% reportó otro nivel diferente al señalado en la encuesta, además de que más de la mitad (64\%) afirma que trabaja, ver Tabla 1.

Tabla 1. Características generales de la población de estudio.

\begin{tabular}{lll} 
Variable & & N (\%) \\
\hline \hline Sexo & & \\
\hline & Femenino & $311(50.7 \%)$ \\
& Masculino & $303(49.3 \%)$ \\
\hline Nivel de estudios & & \\
\hline & Secundaria & $57(9.3 \%)$ \\
& Preparatoria & $171(27.9 \%)$ \\
& Licenciatura & $348(56.7 \%)$ \\
& Posgrado & $26(4.2 \%)$ \\
& Otro & $12(2 \%)$ \\
\hline Trabaja & & \\
\hline & Sí & $393(64 \%)$ \\
& No & $221(36 \%)$ \\
\hline
\end{tabular}




\begin{tabular}{lll}
\hline Edad & & \\
\hline 18 a 24 & $339(55.25 \%)$ \\
25 a 34 & $150(24.4 \%)$ \\
35 a 44 & $57(9.3 \%)$ \\
45 a 54 & $53(8.65 \%$ \\
55 y más & $15(2.45 \%$ \\
\hline
\end{tabular}

Fuente: Elaboración propia.

\subsection{Información sobre el tiempo, días y horas que le dedican a la actividad física agrupada por sexo}

En este estudio se encontró una asociación entre el sexo de las personas y el tiempo que llevan haciendo AF (Chicuadrada $=17.74, \mathrm{p}=0.001$ ). Como se muestra en la Tabla 2 , los hombres tienden a tener más tiempo realizando AF (más de 3 años), y en cambio, las mujeres son las que tienen menos (1 a 6 meses). También, se encontró una asociación entre la frecuencia semanal que realizan $\mathrm{AF}$ (Chi- cuadrada $=12.861, \mathrm{P}=0.002$ ) y el tiempo que le dedican al día (Chi-cuadrada $=13.53, \mathrm{p}=0.001$ ). La asistencia más frecuente fue de 4 a 5 veces por semana (52.9\%), siendo mayor el porcentaje de mujeres (55\%) que de hombres (50.8\%). La frecuencia diaria (de 6 a 7 veces a la semana) la reportan con mayor ocurrencia los hombres, y el tiempo que le dedican al día regularmente es de una hora (47.2\%), mientras que la frecuencia es más recurrente en las mujeres (54.3\%), puesto que los hombres dedican más tiempo.

Tabla 2. Tiempo, días y horas que le dedican a la actividad física agrupada por sexo.

\begin{tabular}{lllll|}
\hline \multirow{2}{*}{$\begin{array}{l}\text { Tiempo practicando } \\
\text { actividad física }\end{array}$} & \multicolumn{2}{|l|}{ Sexo } & \\
\cline { 2 - 4 } & Femenino & Masculino & $\%$ \\
\hline Menos de un mes & $19.3 \%$ & $9.9 \%$ & $14.7 \%$ \\
\hline 1 a 6 meses & $28.9 \%$ & $23.8 \%$ & $26.4 \%$ \\
\hline Más de 6 a 12 meses & $13.5 \%$ & $16.2 \%$ & $14.8 \%$ \\
\hline Más de 12 a 3 años & $18.0 \%$ & $20.1 \%$ & $19.1 \%$ \\
\hline Más de 3 años & $20.3 \%$ & $30.0 \%$ & $25.1 \%$ \\
\hline \hline
\end{tabular}

\begin{tabular}{llll}
\hline $\begin{array}{l}\text { Veces que acude a la } \\
\text { semana }\end{array}$ & $\begin{array}{l}\text { Femenino } \\
36.3 \%\end{array}$ & $\begin{array}{l}\text { Masculino } \\
30.7 \%\end{array}$ & \begin{tabular}{l}
$33.6 \%$ \\
\hline 1 a 3 veces
\end{tabular} \\
\hline 4 a 5 veces & $55.0 \%$ & $50.8 \%$ & $52.9 \%$ \\
\hline 6 a 7 veces & $8.7 \%$ & $18.5 \%$ & $13.5 \%$ \\
\hline \hline $\begin{array}{l}\text { Tiempo diario que le } \\
\text { dedica }\end{array}$ & Femenino & Masculino $\%$ & $\%$ \\
\hline $\begin{array}{l}1 \text { hora } \\
2 \text { horas }\end{array}$ & $54.3 \%$ & $39.9 \%$ & $47.2 \%$ \\
Más de 2 horas & $36.0 \%$ & $44.9 \%$ & $40.4 \%$ \\
\hline Fuente: Elaboración propia. & $9.6 \%$ & $15.2 \%$ & $12.4 \%$ \\
\hline
\end{tabular}

\subsection{Información sobre el tiempo, días y horas que le dedican a la actividad física agrupada por rango de edad}

La variable edad fue categorizada según la clasificación hecha por INEGI, en cinco grupos, tal y como se observa en la Tabla 3. No se encontró diferencias significativas entre el tiempo que llevan practicando actividad física y el rango de edad ( $\mathrm{p}=0.191)$. La mayoría afirma que lleva de uno a seis meses realizando AF, a excepción de los sujetos de 45 a 54 años, en donde el $43.4 \%$ menciona que tiene más de 3 años. En cuanto a la frecuencia semanal, la mayoría asiste de 4 a 5 veces a la semana. Se encontró una asociación negativa leve, pero relevante ( $t a u-b$ de Kendall $=-0.08, p=0.030$ ), $\mathrm{y}$ que conforme aumenta la edad, disminuyen los días semanales de AF. Este mismo comportamiento se observa para la variable: horas dedicadas a la actividad física (tau- $b$ de Kendall $=-0.22$, $P<0.05$ ), cuyo tiempo regular es de una hora, pero se observa que son los jóvenes los que mayor tiempo dedican a la actividad física y, como ya se mencionó, conforme aumenta la edad, disminuye dicho tiempo en horas. 
Tabla 3. Tiempo, días y horas que llevan practicando actividad física agrupada por rango de edad.

\begin{tabular}{|c|c|c|c|c|c|c|}
\hline \multirow{2}{*}{$\begin{array}{l}\text { Tiempo practicando AF } \\
18 \text { a } 24\end{array}$} & \multicolumn{5}{|c|}{ Rango de edad } & \\
\hline & 25 a 34 & 35 a 44 & 45 a 54 & 55 y más & $\%$ & \\
\hline Menos de un mes & $14.5 \%$ & $16.7 \%$ & $19.3 \%$ & $5.7 \%$ & $13.3 \%$ & $14.7 \%$ \\
\hline 1 a 6 meses & $26.3 \%$ & $29.3 \%$ & $22.8 \%$ & $20.8 \%$ & $33 \cdot 3 \%$ & $26.4 \%$ \\
\hline Más de 6 a 12 meses & $15.6 \%$ & $14.0 \%$ & $17.5 \%$ & $11.3 \%$ & $6.7 \%$ & $14.8 \%$ \\
\hline Más de 12 a 3 años & $17.4 \%$ & $22.7 \%$ & $17.5 \%$ & $18.9 \%$ & $26.7 \%$ & $19.1 \%$ \\
\hline Más de 3 años & $26.3 \%$ & $17.3 \%$ & $22.8 \%$ & $43.4 \%$ & $20 \%$ & $25.1 \%$ \\
\hline Días a la semana & 18 a 24 & 25 a 34 & 35 a 44 & 45 a 54 & 55 y más & $\%$ \\
\hline 1 a 3 veces & $32.4 \%$ & $32.0 \%$ & $31.6 \%$ & $41.5 \%$ & $53.3 \%$ & $33.6 \%$ \\
\hline 4 a 5 veces & $50.1 \%$ & $59 \cdot 3 \%$ & $59.6 \%$ & $49.1 \%$ & $40.0 \%$ & $52.9 \%$ \\
\hline 6 a 7 veces & $17.4 \%$ & $8.7 \%$ & $8.8 \%$ & $9.4 \%$ & $6.7 \%$ & $13.5 \%$ \\
\hline Horas diarias & 18 a 24 & 25 a 34 & 35 a 44 & 45 a 54 & 55 y más & $\%$ \\
\hline 1 hora & $38.3 \%$ & $51.3 \%$ & $63.2 \%$ & $62.3 \%$ & $93.3 \%$ & $47.2 \%$ \\
\hline 2 horas & $44.0 \%$ & $42.7 \%$ & $33.3 \%$ & $28.3 \%$ & $6.7 \%$ & $40.4 \%$ \\
\hline Más de 2 horas & $17.7 \%$ & $6.0 \%$ & $3.5 \%$ & $9.4 \%$ & $\mathrm{o} \%$ & $12.4 \%$ \\
\hline
\end{tabular}

Fuente: Elaboración propia.

\subsection{Motivos de la práctica de la actividad física}

La versión traducida de la escala MPAM-R aplicada a esta población mostró una alta consistencia interna (0.949) sin eliminar ningún ítem, siendo para los factores todas superiores a 0.80. Para el factor disfrute se obtuvo un alpha de 0.87; para el de apariencia de 0.83, el social de 0.86 , fitness/salud de 0.84 y el factor competencia de 0.89 .

Los puntajes medios más altos fueron para el ítem 23 "Porque quiero mantener mi salud física y mi bienestar" con un valor de 6.41; el ítem 1 "Realizo actividad física porque quiero estar en buena forma”, con media 6.36, y el ítem 19 "Porque quiero mantener mi fuerza física para vivir de forma saludable”, con valor medio de 6.29, todos pertenecientes al factor fitness/salud. En cambio, los ítems con valores medios más bajos fueron el 28 "Para buscar la aceptación de los demás" y 27 "Porque me sentiré poco atractivo físicamente si no lo practico" con medias 3.72 y 4.58 , respectivamente. El primero hace referencia a lo social y el puntaje medio se ubica en una respuesta "neutra"; mientras el segundo es para el factor apariencia y tiene un puntaje medio en la escala de "ligeramente de acuerdo".

En la Tabla 4, se reportan las medidas descriptivas para cada factor agrupadas por sexo y para el total de la muestra. Se observa que el factor con puntaje medio más alto para toda la muestra es el fitness o salud $(6.25 \pm 0.90)$ seguido del factor disfrute $(5.75 \pm 1.07)$. Aunque no se encontraron diferencias significativas ( $p>0.05$ ) en estos factores en función del sexo, se observa que las mujeres reportan puntajes medios más altos en el factor salud (6.32 \pm 0.71 ), comparado a los hombres, que puntúan más alto en el factor disfrute $(5.77 \pm 1.18)$. El factor con menor puntaje fue el social (4.74 \pm 1.50$)$, siendo los hombres en este caso los que obtienen valores más elevados. Se encontraron, también, diferencias significativas entre sexo únicamente en el factor competencia ( $\mathrm{p}=0.01)$, siendo los hombres los que superan el puntaje medio. 
Tabla 4. Puntaje medio, desviación estándar y p valor para cada factor agrupado por sexo y para toda la muestra.

\begin{tabular}{|c|c|c|c|c|}
\hline & Femenino & Masculino & $\mathrm{p}$ & Muestra \\
\hline Factor & Media $\pm \mathrm{DE}$ & Media \pm DE & $\mathrm{P}$ & Media \pm DE \\
\hline Disfrute & $5.73 \pm 0.95$ & $5.77 \pm 1.18$ & 0.113 & $5.75 \pm 1.07$ \\
\hline Apariencia & $5.59 \pm 1.02$ & $5.44 \pm 1.29$ & 0.495 & $5.52 \pm 1.16$ \\
\hline Social & $4.63 \pm 1.53$ & $4.86 \pm 1.47$ & 0.053 & $4.74 \pm 1.50$ \\
\hline Fitness/salud & $6.32 \pm 0.71$ & $6.18 \pm 1.06$ & 0.535 & $6.25 \pm 0.90$ \\
\hline Competencia & $5.62 \pm 1.03$ & $5.75 \pm 1.20$ & 0.01 & $5.69 \pm 1.12$ \\
\hline
\end{tabular}

Fuente: Elaboración propia. DE = Desviación estándar.

\subsection{Relación entre los motivos de la práctica de la actividad física y la edad}

Con respecto a la edad, los resultados muestran diferencias significativas entre los factores apariencia $(\mathrm{p}=0.019)$ y salud ( $\mathrm{p}=0.036)$. Según la prueba realizada post-hoc de Tukey, para el primer caso, la apariencia es un motivo más importante para el grupo de edad de 25 a 34 en comparación con el grupo de 45 a 54 años ( $\mathrm{p}=0.019)$. En cambio, en el factor salud, la misma prueba post-hoc mostró que la salud es más significativa para realizar AF conforme aumenta la edad. La diferencia se marcó entre el grupo de 18 a 24 años, cuyo puntaje medio fue de 6.18, contra el grupo de 35 a $44(\mathrm{p}=0.038)$ y el de 45 a 54 ( $\mathrm{p}=0.034)$, ver Tabla 5. En este factor se encontró una relación positiva significativa $(\mathrm{p}<0.01)$ con la edad, lo cual nos sugiere que, a mayor edad, los motivos de salud para esta población son los más influyentes en su decisión para realizar AF.

Tabla 5. Puntaje medio y desviación estándar de cada factor en cada uno de los grupos de edad (años).

\begin{tabular}{|c|c|c|c|c|c|c|}
\hline \multirow[b]{2}{*}{ Factor } & \multicolumn{5}{|c|}{ Grupo de edad } & \\
\hline & 18 a 24 & 25 a 34 & 35 a 44 & 45 a 54 & 55 y más & \\
\hline & Media $\pm \mathrm{DE}$ & Media $\pm \mathrm{DE}$ & Media $\pm \mathrm{DE}$ & Media $\pm \mathrm{DE}$ & Media $\pm \mathrm{DE}$ & $\mathrm{p}$ \\
\hline Disfrute & $5.68 \pm 1.04$ & $5.83 \pm 1.08$ & $5.95 \pm 1.04$ & $5.66 \pm 1.22$ & $5.96 \pm 0.88$ & 0.261 \\
\hline Apariencia & $5.45 \pm 1.19$ & $5.72 \pm .99$ & $5.74 \pm 1.10$ & $5.15 \pm 1.35$ & $5.58 \pm 1.17$ & 0.019 \\
\hline Social & $4.66 \pm 1.44$ & $4.87 \pm 1.45$ & $5.06 \pm 1.60$ & $4.42 \pm 1.84$ & $5.25 \pm 1.47$ & 0.062 \\
\hline Fitness/salud & $6.18 \pm 0.92$ & $6.25 \pm 0.97$ & $6.45 \pm 0.89$ & $6.49 \pm 0.55$ & $6.47 \pm 0.48$ & 0.036 \\
\hline Competencia & $5.71 \pm 1.08$ & $5.72 \pm 1.09$ & $5.76 \pm 1.19$ & $5.42 \pm 1.31$ & $5.62 \pm 1.28$ & 0.465 \\
\hline
\end{tabular}

$\mathrm{DE}=$ desviación estándar

Fuente: Elaboración propia. 


\subsection{Relación entre los motivos de la práctica de la actividad física y tiempo que llevan practicando}

La Tabla 6, reporta los puntajes medios de cada factor según el tiempo que llevan practicando la actividad física, y se encontraron diferencias significativas en todos los factores excepto en el social. Para el caso del factor disfrute, la prueba de post-hoc mostró diferencias entre los que tienen menos de un mes practicando AF contra los grupos que tienen de más de seis meses ( $\mathrm{p}<0.05)$. Los que tienen más de un año hasta tres son reportados con los valores medios más altos en este factor, mientras que los de menor tiempo, valores medios más bajos. En otras palabras, entre más tiempo llevan practicando actividad física, más es el disfrute por la actividad. Por su parte, en el factor apariencia, la discrepancia se encontró entre los grupos de menos de un mes practicando, frente a los de uno a seis meses $(\mathrm{p}=0.015)$, más de un año a tres
( $\mathrm{p}=0.001)$ y los de más de tres años $(\mathrm{p}=0.045)$. En este estudio, el puntaje medio más alto en apariencia se encontró en los que llevan más de tres años practicando actividad física. En lo que respecta al factor salud, la diferencia la marcó el grupo que llevaba practicando menos de un mes $(\mathrm{p}<0.05)$. Los motivos relacionados con la salud fueron iguales entre los que tenían de uno a seis meses con los de más de seis meses a un año (p $>0.05$ ). De igual forma, entre los que tenían más de un año a tres, con los de más de tres años ( $\mathrm{p}>0.05)$. Los puntajes medios relacionados con la salud fueron más altos en los que tenían más de un año a 3 y más bajos en los de menor tiempo practicando AF.

En general, entre más tiempo llevan practicando actividad física, más altos son los puntajes de disfrute, apariencia, fitness/salud y competencia.

Tabla 6. Puntaje medio y desviación estándar de los motivos de la práctica de la AF según el tiempo que llevan practicándola.

\begin{tabular}{|c|c|c|c|c|c|c|}
\hline \multirow{2}{*}{ Motivos } & \multicolumn{5}{|c|}{ Tiempo practicando la actividad física } & \\
\hline & Menos de un mes & 1 a 6 meses & $\begin{array}{l}\text { Más de } 6 \text { a } 12 \\
\text { meses }\end{array}$ & $\begin{array}{l}\text { Más de } 12 \text { a } 3 \\
\text { años }\end{array}$ & Más de 3 años & \\
\hline & Media $\pm \mathrm{DE}$ & Media $\pm \mathrm{DE}$ & Media $\pm \mathrm{DE}$ & Media $\pm \mathrm{DE}$ & Media $\pm \mathrm{DE}$ & $\mathrm{p}$ \\
\hline Disfrute & & & $5.76 \pm 0.92$ & $6.09 \pm 0.86$ & & 0.000 \\
\hline Apariencia & $5.11 \pm 1.33$ & $5 \cdot 58 \pm 1.16$ & $5 \cdot 50 \pm 1.11$ & $5.74 \pm 1.08$ & $5.53 \pm 1.09$ & 0.005 \\
\hline Social & $4.41 \pm 1.43$ & $4.75 \pm 1.56$ & $4.76 \pm 1.43$ & $4.98 \pm 1.61$ & $4.75 \pm 1.42$ & 0.067 \\
\hline Fitness/salud & $5.87 \pm 1.05$ & $6.20 \pm 0.95$ & $6.19 \pm 0.92$ & $6.55 \pm 0.54$ & $6.33 \pm 0.88$ & 0.000 \\
\hline Competencia & $5 \cdot 38 \pm 1.11$ & $5 \cdot 54 \pm 1.25$ & $5 \cdot 59 \pm 1.12$ & $6.00 \pm 0.92$ & $5.84 \pm 1.05$ & 0.000 \\
\hline
\end{tabular}

DE= desviación estándar

Fuente: Elaboración propia. 


\subsection{Relación entre los motivos de la práctica de la actividad física y días que acuden a la semana a realizarla}

En la Tabla 7, se muestran los valores medios para cada uno de los factores según los días a la semana y horas diarias que practican actividad física. Se encontraron diferencias significativas únicamente en los factores de apariencia, salud y competencia para los días de la semana que le dedican a la AF. Para cada uno de estos factores, estas diferencias fueron entre los grupos que acuden de 1 a 3 veces a la semana, frente a los que van de 4 a 5 (p $<0.05)$. En cuanto a la variable "horas que le dedican a la actividad física diariamente", solo se detectaron diferencias ( $p=.029$ ) en el factor competencia. Asimismo, se puede observar en la Tabla 7 que en los que practican más de 2 horas, el puntaje en el factor competencia es mayor comparado con los que practican menos tiempo.

Tabla 7. Puntaje medio y desviación estándar de los motivos de la práctica de la AF según días a la semana que la realizan y horas diarias

\begin{tabular}{|c|c|c|c|c|}
\hline \multirow{3}{*}{ Motivos } & \multicolumn{4}{|c|}{ Días a la semana que hace $\mathrm{AF}$} \\
\hline & 1 a 3 & 4 a 5 & 6 a 7 & \\
\hline & Media \pm DE & Media \pm DE & Media $\pm \mathrm{DE}$ & $\mathrm{p}$ \\
\hline Disfrute & $5.66 \pm 0.95$ & $5.80 \pm 1.07$ & $5.74 \pm 1.30$ & 0.329 \\
\hline Apariencia & $5.36 \pm 1.72$ & $5.62 \pm 1.12$ & $5 \cdot 51 \pm 1.26$ & 0.036 \\
\hline Social & $4.77 \pm 1.49$ & $4.72 \pm 1.50$ & $4.75 \pm 1.58$ & 0.924 \\
\hline Fitness /salud & $6.11 \pm 0.88$ & $6.36 \pm 0.83$ & $6.16 \pm 1.14$ & 0.000 \\
\hline Competencia & $5.53 \pm 1.01$ & $5.76 \pm 1.12$ & $5.78 \pm 1.34$ & 0.001 \\
\hline \multicolumn{5}{|c|}{ Horas diarias que practica $\mathrm{AF}$} \\
\hline \multirow{2}{*}{ Motivos } & 1 hora & 2 horas & Más de 2 & \\
\hline & Media $\pm \mathrm{DE}$ & Media $\pm \mathrm{DE}$ & Media $\pm \mathrm{DE}$ & $\mathrm{p}$ \\
\hline Disfrute & $5.70 \pm 1.09$ & $5.77 \pm 1.04$ & $5.88 \pm 1.06$ & 0.376 \\
\hline Apariencia & $5.44 \pm 1.22$ & $5.58 \pm 1.12$ & $5.61 \pm 1.08$ & 0.412 \\
\hline Social & $4.72 \pm 1.59$ & $4.12 \pm 1.43$ & $4.92 \pm 1.38$ & 0.307 \\
\hline Fitness & $6.24 \pm 0.82$ & $6.29 \pm 0.95$ & $6.14 \pm 1.06$ & 0.529 \\
\hline Competencia & $5.61 \pm 1.141$ & $5.70 \pm 1.10$ & $5.93 \pm 1.09$ & 0.029 \\
\hline
\end{tabular}

$\mathrm{DE}=$ desviación estándar

Fuente: elaboración propia.

\section{Discusión y conclusiones}

Actualmente, conocer los motivos por los cuales ciertas poblaciones realizan AF ha cobrado interés para algunos investigadores debido a que ciertos atributos de las personas como la edad y el sexo pueden marcar diferencia en las tendencias para la práctica del ejercicio (Jiménez et al., 2012; Azofeifa, 2018). Los resultados de este estudio muestran que los hombres son los que presentan mayores tiempos dedicados a las actividades deportivas, mientras que las mujeres practican AF de manera regular una hora diaria de 4 a 5 veces a la semana. Estos resultados coinciden con otros estudios en poblaciones tanto mexicanas como de otros países, en donde se reporta la escasa participación del sexo femenino en actividades físicas y deportivas (Gómez et al., 2010; Deaner \& Smith, 2012; Mabry et al., 2012; Zamarripa et al., 2013; Li et al., 2015). Sin embargo, esta tendencia no ha sido probada en otros estudios, tal es el caso del realizado por AL-Hazaa (2007) en una población de Arabia Saudita, en donde la 
actividad física fue más activa en las mujeres. De igual forma en el trabajo reportado por Jiménez et al. (2012), en una población de jóvenes españoles, aunque se señala que los hombres realizan más actividad física que las mujeres, no se encontró diferencias significativas en función del sexo en cuanto a la frecuencia semanal. Un factor que puede influir en estos resultados del sexo femenino es que, además de su participación laboral, estas generalmente también realizan actividades relacionadas con el hogar y la familia (quehacer doméstico, el traslado de los hijos a la escuela, etcétera), lo cual conlleva a la falta de tiempo para realizar AF.

Por otro lado, este estudio reporta que son los jóvenes los que asisten con más frecuencia y con más horas de duración en ejercicio físico. Estos resultados son congruentes con los reportados en otros estudios con poblaciones mexicanas, en donde se afirma que los jóvenes son más activos físicamente que los adultos, sobre todo que los adultos mayores (Muñoz et al., 2016). En la misma línea, en el estudio de Zamarripa et al. (2013) se observa que la prevalencia de personas activas va disminuyendo progresivamente a través de los diferentes grupos de menor a mayor edad. Estos resultados podrían atribuirse a la carga laboral de los adultos o situaciones relacionadas con la familia, como también puede ser algún problema de salud, que les impida realizar ejercicio. Los jóvenes, mientras tienen más tiempo disponible para actividades recreativas, entre ellas el deporte.

Así, el instrumento utilizado en este estudio para medir los motivos de la AF reporta niveles de confiabilidad superiores a otros estudios (Moreno et al., 2007; Granero et al., 2011;) en todas las subescalas, a excepción del factor apariencia. De igual forma, en poblaciones con características similares a la mexicana se reportaron valores más altos de confiabilidad en la escala de Disfrute, Fitness/salud y Competencia (Celis-Merchán, 2006). En la población estudiada, se encontró que los motivos más importantes para hacer AF tanto en hombres como en las mujeres encuestadas son la salud y el disfrute por la actividad, siendo lo menos relevante las relaciones sociales, como la convivencia con los amigos. Se destaca el hecho, a su vez, de que en las mujeres es más determinante la salud y enlos hombres el disfrute. En cuanto a este factor de salud, cabe destacar, y considerando los datos de la OMS (2010), que la inactividad física es considerada como la principal causa en casi la cuarta parte de los casos de cáncer de colon, en el $27 \%$ de los casos de diabetes y en casi un 30\% de las cardiopatías, razón por la cual se recomienda hacer AF por lo menos 60 minutos o un poco más de acuerdo con la edad. Los presentes resultados así mismo coinciden con los de Moreno et al. (2007), donde para los factores de apariencia y salud las mujeres fueron las que presentaron mayores valores. No así, el de Granero et al. (2011) muestra como factor con mayor puntaje el social.

Si bien los motivos para ejercitarse son variados, la preocupación por la salud ha sido una constante en muchas investigaciones realizadas en esta línea (Sicilia et al., 2014; Muñoz et al., 2016; Azofeifa, 2018), y más aun en las mujeres (Li et al., 2015; Tylka \& Homan, 2015).

En este estudio no se encontraron diferencias significativas para cada una de las subescalas del instrumento, a excepción del factor competencia, en donde los hombres reportan valores medios más altos que las mujeres, dato que concuerda también con otras poblaciones (Cagas et al., 2010; Guedes et al., 2013), en donde el sexo femenino tienden a ejercitarse más por cuestiones estéticas y los hombres por atributos asociados con el desarrollo de habilidades de su cuerpo y a actividades que suponen un reto físicamente.

Otro atributo importante que se tomó en cuenta fue la edad, donde se observan cambios significativos en el factor apariencia y salud, destacando el hecho de que a los jóvenes los motiva más la apariencia y lo estético, sobre todo en mujeres, mientras que la prevención de enfermedades es el factor determinante para los de mayor edad., hallazgos que coinciden con otros estudios, en donde se manifiesta que regularmente las personas mayores de 40 años se preocupan más por su salud y el control del peso corporal, mientras que los jóvenes atribuyen la AF de manera significativa a motivos de diversión o enfrentar desafíos y/o el gusto por competir (Moreno et al., 2007; Guedes et al., 2013; Sicilia et al., 2014). Este comportamiento puede deberse al hecho de que los jóvenes no son conscientes aún de los beneficios 
que el ejercicio puede aportar a su salud, puesto que las enfermedades se presentan con mayor frecuencia en edades más avanzadas. Pese a esto, hay poblaciones en donde las personas de mayor edad tienden a darle más importancia a los motivos de competencia para justificar su actividad física, como es el caso del reportado por Frederick \& Schuster-Smith (2003).

Ahora bien, se halló una relación entre los motivos por los cuales las personas hacen actividad física y el tiempo que llevan realizándola, puesto que las personas que mayor experiencia poseen en la práctica del ejercicio, reportan mayores niveles de disfrute, salud, apariencia y competencia. Estos datos concuerdan con otras investigaciones realizadas, en las que se reporta que las personas con más años en el ejercicio físico presentan valores más elevados de disfrute (Guedes et al., 2013; Azofeifa, 2018). De igual manera, en el presente estudio se encontró que el mantener una buena imagen, salud y el desarrollo de habilidades lleva a las personas a realizar ejercicio con mayor frecuencia a la semana, sin embargo, únicamente los motivos de competencia impulsan a los participantes a durar más tiempo en la actividad.

Se concluye, entonces, que conocer los principales motivos por los cuales ciertos sectores de la población realizan actividad física es fundamental, ya que permite diseñar estrategias orientadas a estos grupos, poniendo a su disposición información relevante dirigida a fomentar o continuar la práctica de AF. Afortunadamente, se encuentra en la literatura bastante información que pone de manifiesto los grandes beneficios del ejercicio, como disminuir los índice de mortalidad asociadas a las ECNG que se derivan del sedentarismo, por ejemplo. En esta población específica, se observó que los motivos que llevan a las personas a realizar actividad física son factores extrínsecos, especialmente los que tienen que ver con la salud y la apariencia, sobre todo en poblaciones de mujeres en cualquier rango de edad. En cambio, los hombres, aunque dan importancia a la salud, sobre todo los de mayor edad, se ven más motivados por características intrínsecas.

Finalmente, en cuanto a las limitaciones que podemos destacar en este estudio, existe la condición del tamaño de la muestra, donde esta no fue tomada de manera aleatoria.
Se considera que para futuros estudios en esta misma línea de investigación, podría procederse con una muestra de mayor tamaño, tomando en cuenta la dimensión de la población que realiza actividad física. A su vez, otra limitante fue el tiempo disponible por los encuestados, ya que pese a que hubo buena disposición por parte de los entrevistados, la mayoría tuvo que disponer de su tiempo de ejercicio para contestar la encuesta, por lo que algunas preguntas fueron contestadas de manera muy rápida. No obstante, los resultados obtenidos en esta investigación se asemejan a los reportados en otros estudios en poblaciones mexicanas y de otra nacionalidad.

\section{Referencias bibliográficas}

Al-Hazzaa, H. (2007). 'Health-enhancing physical activity among Saudi adults using the International Physical Activity Questionnaire (IPAQ)'. Public Health Nutrition, 10(01), p59-64 [en línea]. Disponible en: https://doi.org/10.1017/ S1368980007184299

Azofeifa, C.A. (2018). 'Comparación entre los motivos para la práctica de actividad física según el género y el tiempo de práctica en un grupo de estudiantes'. MHSalud, 14(2) [en línea]. Disponible en: https:// doi.org/10.15359/mhs.14-2.1

Cagas, J. Y., Torres, B., \& Manalastas, E.J. (2010). ‘'Why do Filipinos exercise? Exploring motives from the perspective of Filipinos youth'. In: Chia M. Wang J, Balasekaran G, Chatzisarantis N (Eds). Proceeding of the III International Conference of Physical Education and Sports Science, p243248. Singapore: National Institute of Education.

Cambronero, M., Blasco, J.E., Chiner, E., Lucas-Cuevas, A.G. (2015). 'Motivos de participación de los estudiantes universitarios en actividades físicodeportivas'. Revista Iberoamericana de Psicología del Ejercicio y el Deporte, 10(2), p179-186 [en línea]. Disponible en: http://www.redalyc.org/ articulo.oa?id=311137747002 
Celis_Marchán, A.G. (2006). 'Adaptación al español de la escala revisada de motivos para la actividad física (MPAM-R) y el cuestionario de clima deportivo (SCQ)'. Avances en Medicina, 4, p73-9o [en línea]. Disponible en: http://www.humanas.unal.edu.co/ psicometria/files/7613/7036/4859/Adaptacin_Al_ Espaol_De_La_Escala_Revisada_De_Motivos_ Para_La_Actividad_Fsica_MPAM-R_Y_El_ Cuestionario_De_Clima_Deportivo_SCQ.pdf

Deaner, R. \& Smith, B. (2012). 'Sex Differences in sports across 50 societies'. Peer Reviewed Articles, 2, p140 [en línea]. Disponible en https://scholarworks. gvsu.edu/psy_articles/2

Deci, E. L. \& Ryan, R. M. (1985). 'The general causality orientations scale: Self- determination in personality'. Journal of Research in Personality, 19(2), p109-134 [en línea]. Disponible en: https:// doi.org/10.1016/oo92-6566(85)90023-6

Department of Health and Human Services. (2018). 2018 Physical Activity Guidelines Advisory Committee. Washington, DC: U'. Department of Health and Human Services. Disponible en: https://health. gov/paguidelines/second-edition/report/pdf/PAG_ Advisory_Committee_Report.pdf [5 febrero 2019].

Encuesta Nacional de Salud y Nutrición. (2016). Disponible en: https://ensanut.insp.mx/ensanut2016/index. php\#.XFHdHVxKiUk [19 febrero 2019].

Frederick, C.M., \& Schuster, H. (2003). 'Competition and intrinsic motivation in physical activity: A comparison of two groups'. Journal of Sport Behavior, 26(3), p240-254 [en línea]. Disponible en: https:// selfdeterminationtheory.org/SDT/documents/2003 FrederickRecSchusterSmi_JSB.pdf

George, D. \& Mallery, P. (2003). SPSS for Windows step by step: A Simple Guide and Reference. $4 .{ }^{\mathrm{a}} \mathrm{ed}$. Boston: Allyn \& Bacon [en línea]. Disponible en: https://wps.ablongman.com/wps/media/ objects/385/394732/george4answers.pdf
Gómez, M., Ruiz, F \& García M.E. (2010). 'Actividades físicodeportivas que demandan los Universitarios'. Espiral. Cuadernos del profesorado, 3(5), p3-10 [en línea]. Disponible en: https://dialnet.unirioja. es/servlet/articulo?codigo $=3160549$

Granero-Gallegos, A., Gómez-López, M., Abraldes, J.A \& Rodríguez, N. (2011). 'Motivos de práctica en el ámbito de la actividad física no competitiva'. Espiral, Cuadernos del profesorado, 4(7), p1522 [en línea]. Disponible en: https://core.ac.uk/ download/pdf/154261910.pdf

Guedes, D.P., Legnani, R.F.S., Legnani, E. (2013). ‘Exercise motives in a sample of Brazilian university students'. Motriz. Rio Claro, 19(3), p590-596 [en línea]. Disponible en: http://www.scielo.br/pdf/ motriz/v19n3/og.pdf

Instituto Nacional de Estadística y Geografía (INEGI). (2018). Disponible en: https://www.inegi.org.mx/ app/saladeprensa $/$ noticia.html $\mathbf{i d}=4722$

Jiménez, M.G., Godoy, D. \& Godoy, G. (2012). 'Relación entre los motivos para la práctica físico-deportiva y las experiencias de flujo en jóvenes: diferencias en función del sexo'. Universitas Psychologica, 11(3), p9o9-920 [en línea]. Disponible en: https://revistas.javeriana. edu.co/index.php/revPsycho/article/view/9o9

Li, C., Zayed, K., Muazzam, A., Li, M., Cheng, J., \& Chen, A. (2015). 'Motives for Exercise in Undergraduate Muslim Women and Men in Oman and Pakistan Compared to the United States'. Sex Roles, 72(12), p68-84 [en línea]. Disponible en: https://link. springer.com/article/10.1007/s11199-014-0435-Z

Mabry, R.M., Winkler, E.A., Reeves, M.M., Eakin, E.G., \& Owen, N. (2012). 'Associations of physical activity and sitting time with the metabolic syndrome among Omani adults'. Obesity, 20(11), p2290-2295 [en línea]. Disponible en: https://doi.org/10.1038/oby.2012.26 
Moreno, J.E \& Cruz, H.F. (2015). 'Asociación entre los motivos y la práctica de ejercicio en participantes de un programa de actividad física laboral'. Rev. Fac. Med, 63(4), p6o9-615 [en línea]. Disponible en: https://doi.org/10.15359/mhs.14-2.1

Moreno, J.A, Marcos, P.J., Huéscar, E. (2016). 'Motivos de práctica Físico-Deportiva en mujeres: Diferencias entre practicantes y no practicantes'. Revista de Psicología del Deporte, 25(1), p35-41 [en línea]. Disponible en: https://www.rpd-online.com/ article/view/v25-n1-moreno-murcia-marcos-etal

Moreno, J.M., Cervelló, G. \& Martínez, A. (2007). 'Validación de la Escala de Medida de los Motivos para la Actividad Física-Revisada en españoles: Diferencias por motivos de participación'. Anales de psicología, 23(1), p167-176 [en línea]. Disponible en: http://www.redalyc.org/articulo.oa?id=16723120

Muñoz-Daw, M.J., Muñoz-Duarte, M., De La Torre-Díaz, M.L., Hinojos-Seáñez, E., \& Pardo-Rentería, J.B. (2016). 'Motivos para la práctica de actividad física recreativa e inactividad en la población de Chihuahua (México)'. Nutrición Clínica Dietética Hospitalaria, 36(1), p10-16 [en línea]. Disponible en: https:// dialnet.unirioja.es/servlet/articulo?codigo $=5405198$

Organización Mundial de la salud. (2010). Disponible en: http://www.who.int/dietphysicalactivity/pa/es/ [15 marzo 2019].

Pérez, S. \& Sánchez, M. (2015). 'Motivos de participación y satisfacción en actividades cooperativas y no competitivas en el medio natural'. Ágora para la Educación Física y el Deporte, 17(3), p284-299 [en línea]. Disponible en: http://agora-revista.blogs.uva. es/files/2015/12/agora_17_3f_perez_et_sanchez.pdf

Ryan, R.M., Frederick, C.M., Lepes, D., Rubio, N. \& Sheldon, K.M. (1997). 'Intrinsic motivation and exercise adherence'. International Journal of Sport Psychology, 28, p335-354 [en línea]. Disponible en: https://selfdeterminationtheory. or g / S D T / d o c u m e n t s / 1997 _ RyanFrederickLepesRubioSheldon.pdf
Sanclemente, Y.A. \& Arias, M.N. (2014). 'Factores motivacionales que influyen en la adherencia a la actividad física en los usuarios del Gimnasio Zona Fitness'. Revista de Educación Física, 3(1), p89-116 [en línea]. Disponible en: http://aprendeenlinea. udea.edu.co/revistas/index.php/viref/article/ view/19050

Sicilia, A., González, D., Artes, E.M., Casimiro, A.J., \& Ferriz, R. (2014). 'Motivos de los ciudadanos para realizar ejercicio físico: un estudio desde la teoría de la autodeterminación'. Revista Latinoamericana de Psicología, 46(2) p83-91 [en línea]. Disponible en: https://doi.org/10.1016/ S0120-0534(14)70011-1

Tylka, T. \& Homan, K. (2015). 'Exercise motives and positive body image in physically active college women and men: Exploring an expanded acceptance model of intuitive eating'. Body Imagen, 15, p9o-97 [en línea]. Disponible en: https://doi.org/10.1016/j.bodyim.2015.07.003

Valdés, H. M. (1996). La preparación psicológica del deportista: Mente y rendimiento humano. $3^{\circ} \mathrm{Ed}$. España: INDE publicaciones, p54-57.

Zamarripa, J.I., Ruiz-Juan, F., López J.M., \& Fernández, R. (2013). 'Actividad e inactividad física durante el tiempo libre en la población adulta de Monterrey (Nuevo León México)'. RETOS: Tendencias en Educación Física, Deporte y Recreación, (24), p91-96 [en línea]. Disponible en: http://www. redalyc.org/articulo.oa?id=345732290019 